\title{
Global controllability properties for the semilinear heat equation with superlinear term.
}

\author{
A.Y. KHAPALOV
}

\begin{abstract}
We discuss several global approximate controllability properties for the semilinear heat equation with superlinear reactionconvection term, governed in a bounded domain by locally distributed controls. First, based on the asymptotic analysis in vanishing time, we study the steering of the projections of its solution on any finite dimensional space spanned by the eigenfunctions for the truncated linear part. We show that, if the control-supporting area is properly chosen, then they can approximately be controlled globally at any time in the topology induced by $L^{2}(\Omega)$. Then, based on the $L^{2}\left(Q_{T}\right)$-estimates as $T \rightarrow 0$ for the control functions solving the first problem, we prove that its global approximate controllability from any $u_{0} \in L^{2}(\Omega)$ is also possible at any time in certain topology, which is weaker than that of $L^{2}(\Omega)$. (It is known that this result does not hold in $L^{2}(\Omega)$.) Finally, based on Altman's fixed point theorem and some of the above asymptotictype results, we show that if the nonlinearity is purely of reaction type and is locally Lipschitz, then the global exact controllability in finite dimensions holds as well.
\end{abstract}

\section{Introduction}

\subsection{Problem background}

We consider the following homogeneous Dirichlet problem for the semilinear heat equation:

AMS Subject Classification: 35,93 .

Servicio Publicaciones Univ. Complutense. Madrid, 1999. 


$$
\begin{gathered}
\frac{\partial u}{\partial t}=\Delta u-f(x, t, u, \nabla u)+v(x, t) \chi_{\Omega} .(x) \text { in } Q_{T}=\Omega \times(0, T), v \in L^{2}\left(Q_{T}\right) \\
u=0 \quad \text { in } \Sigma_{T}=\partial \Omega \times T,\left.\quad u\right|_{t=0}=u_{0} \text { in } \Omega .
\end{gathered}
$$

Here $\Omega$ is a bounded domain of an $n$-dimensional Euclidean space $R^{n}$ with boundary $\partial \Omega, \Omega_{*}$ is a Lebesgue's measurable subset of $\Omega$; mes $\left\{\Omega_{*}\right\}>0$, and $\chi \Omega_{*}(x)$ is the characteristic function of $\Omega_{*}$.

In this paper our goal is to study the approximate controllability of (1.1) in the sense, which will be described precisely in the next two subsections. In this subsection we would like to give a brief account of some results available in this area.

Let $H$ be a Banach space, in which, as a phase-space, the boundary problem (1.1) admits a continuous-in-time unique solution. Then, in the classical sense, $(1.1)$ is said to be globally approximately controllable in $H$ at time $T$ if for any given $u_{0} \in H$ the range of its solution mapping at time $T$, namely,

$$
L^{2}\left(Q_{T}\right) \ni v \rightarrow u(\cdot, T) \in H
$$

is dense in $H$ (in the matching topology). One has the exact controllability if this mapping is surjective.

For the case of globally Lipschitz nonlinearity and open $\omega$ this problem was thoroughly studied in the series of works by C. Fabre, J.-P. Puel, E. Zuazua, and L. A. Fernández [2], [4], [15]. The methods of these works are based on the unique continuation property of solutions to a linearized version of (1.1) from an open set, combined with the fixed point argument and the variational approach to the issue of controllability due to J.-L. Lions [10].

For a class of semilinear parabolic equations with varying coefficients and similar globally Lipschitz nonlinearity (but of reaction type only) both the approximate and exact null-controllability (i.e., to an equilibrium) problems were studied by $A$. Fursikov and $O$. Imanuvilov [5]. The method of [5] employs the technique of Carleman's estimates to enhance the above-mentioned unique continuation property.

The success of the above-cited works was essentially due to the possibility to view a nonlinear globally Lipschitz term defined on the solutions 
to the pde at hand as a linear reaction term with uniformly bounded coefficients. For example, if, say, $f=f(u), f(0)=0$, then one can write

$$
\begin{aligned}
f(u(x, t)) & =a(x, t) u(x, t), \quad a \in L^{\infty}\left(Q_{T} \psi, a(x, t)\right. \\
& =\frac{\lfloor(u(x, t))}{u(x, t)}, \quad\|a\|_{L^{\infty}\left(Q_{T}\right) \leq L, \forall u(x, t)}
\end{aligned}
$$

where $L$ is the Lipschitz constant, independent of $x$ and $t$. This allows one to "embed" the semilinear problem at hand into suitably uniformly bounded infinite family of linear ones, thus providing the basis for the fixed point argument. Unfortunately, the situation becomes qualitatively different if one deals with nonlinearity admitting the superlinear growth at infinity, in which case, to our knowledge, fewer results are available.

In the work [3], by E. Fernandez-Cara, both the boundary and internal exact null-controllability, understood in some generalized sense, were shown with $u_{0} \in L^{\infty}(\Omega)$ for the reaction term satisfying the growth rate $\lim _{|p| \rightarrow \infty} f(p) /(p \log |p|)=0$. The method of [3] refines the technique of Carleman's estimates of the work [5] along the lines of the fixed point argument.

In [6], by A. Khapalov, not assuming the uniqueness of a solution, the global approximate controllability of (1.1), (1.2) was shown at any time in $L^{2}(\Omega)$ (in the sense described below in the subsections $1.2,1.3$ ), provided the controls are static: $v=v(x)$ and are supported from the entire $\Omega$. In the one dimensional case it was shown in [7] that, if $|f(x, t, u, \nabla u)| \leq \varphi^{*}(x, t)+\psi(t)\left|f_{*}(x, t, u, \nabla u)\right|$, where $\varphi^{*}(x, t)$ and $f_{*}(x, t, u, \nabla u)$ satisfy the conditions (1.2) below (with $f_{*}$ in place of $f$ ) and $\psi(t) \rightarrow 0$ sufficiently fast as $t$ tends to zero, then (1.1) can be made globally approximately controllable in $L^{2}(\Omega)$ at any time even by means of lumped controls, depending on time only. The method of [6] and [7] does not use the fixed-point argument and is based on the idea to suppress the effect of nonlinearity by using control actions only for asymptotically short time. In [8] the exact null-controllability of $(1,1)$, (1.2) with $f=f(u)$ was shown in $L^{2}(\Omega)$ at any positive time for a class of mobile locally distributed controls, whose support can be made arbitrarily small at every moment of time. 


\subsection{Problem formulation}

In this article we are specifically interested in the study of global approximate controllability of $(1.1)$ in the case when $f$ can grow at a superlinear rate at infinity. ${ }^{-}$More precisely, we assume that $f(x, t, u, p)$ is Lebesgue's measurable in $x, t, u, p$, and continuous in $u, p$ for almost all $(x, t) \in Q_{T}$, and is such that

$$
\begin{gathered}
|f(x, t, u, p)| \leq \varphi^{*}(x, t)+\beta|u|^{q^{*} / q^{\prime}}+\beta\|p\|_{R^{n}}^{m^{*} / q^{\prime}} \\
\text { a.e. in } Q_{T} \text { for } u \in R, p \in R^{n} ; \\
\int_{\Omega} f(x, t, \phi, \nabla \phi) \phi d x \geq(\nu-1) \int_{\Omega}\|\nabla \phi\|_{R^{n}}^{2} d x- \\
\beta(t) \int_{\Omega}\left(1+\phi^{2}\right) d x, \forall \phi \in H_{0}^{1}(\Omega),
\end{gathered}
$$

where $\nu>0, \beta(\cdot) \in L^{1}(0, T), \int_{0}^{T} \beta(t) d t \leq \beta, \varphi^{*} \in L^{q^{\prime}}\left(Q_{T}\right)$, and:

$$
\begin{gathered}
0 \leq q^{*}<q=2+\frac{4}{n}, \quad q^{\prime}=2-\frac{4}{n+4}\left(\frac{1}{q}+\frac{1}{q^{\prime}}=1\right) \\
0 \leq m^{*}<2, m^{*}+q^{*} \neq 0
\end{gathered}
$$

Here and elsewhere we use the standard notations for Sobolev spaces such as $H_{0}^{1,0}\left(Q_{T}\right)=\left\{\phi\left|\phi, \phi_{x_{i}} \in L^{2}\left(Q_{T}\right), i=1, \ldots, n, \phi\right|_{\Sigma_{T}}=0\right\}$ and $H_{0}^{1}(\Omega)=\left\{\phi\left|\phi, \phi_{z i} \in L^{2}(\Omega), i=1, \ldots, n, \phi\right|_{\partial \Omega}=0\right\}$.

Conditions (1.2) ensure the existence of at least one generalized solution of (1.1) from $C\left([0, T] ; L^{2}(\Omega)\right) \cap H_{0}^{1,0}\left(Q_{T}\right)$, which also lies in $L^{q}\left(Q_{T}\right)$ ([9], pp. 466-467). However its uniqueness is not guaranteed. On the other hand, if one assumes in addition to (1.2) that $f$ is locally Lipschitz, say, as in (1.5) below, then it is possible to prove that (1.1) possesses a unique solution.

Having this in mind, it scems natural at the first glance to select $H=L^{2}(\Omega)$ as the space to study the controllability in. However, an example due to $\mathrm{A}$. Bamberger (see also [2], [5], p. 51) indicates that when $f(u)=u|u|^{r}, r>0$, which is a particular case of (1.2), the approximate controllability of $(1.1)$ in any of $L^{p}(\Omega), 1 \leq p<\infty$ is not possible at any positive time. 
From this standpoint, it seems more relevant to ask: Can the global approximate controllability of (1.1), (1.2) be achieved in some weaker than $L^{2}(\Omega)$ topology? The next question arises due to the (rather natural) lack of uniqueness of solutions to (1.1), (1.2): Can we ensure the well-posedness of steering of (1.1), (1.2) when multiple solutions are an option? For example, can we use the same control to steer all the possible multiple solutions (corresponding via (1.1) to it) simultaneously and uniformly to the target state? In this article we answer positively to these questions as follows.

\subsection{Main results}

Denote by $\lambda_{k}, \omega_{k}(x), k=1, \ldots$ the eigenvalues and the orthonormalized in $L^{2}(\Omega)$ eigenfunctions of the spectral problem: $-\Delta \omega=$ $\lambda \omega, \omega \in H_{0}^{1}(\Omega)$. Let $c_{1}, \ldots, c_{k}, \ldots$ be a monotone non-increasing sequence of positive numbers. Denote by $W$ the Banach space of functions $\left\{\phi \mid \phi(x)=\sum_{k=1}^{\infty} \alpha_{k} \omega_{k}(x), \sum_{k=1}^{\infty} \alpha_{k}^{2} c_{k}<\infty\right\}$, endowed with the norm

$$
\|\phi\|_{W}=\left(\sum_{k=1}^{\infty} \alpha_{k}^{2} c_{k}\right)^{1 / 2} \text {. }
$$

Note that, since $L^{2}(\Omega)$ is continuously embedded into $W$, then $u \in$ $C([0, T] ; W)$.

Denote $L_{k}^{2}(\Omega)=\left\{\phi \mid \phi(x)=\sum_{i=1}^{k} \alpha_{i} \omega_{i}(x), \alpha_{i} \in R\right\}$. Define a monotone non-increasing sequence of non-negative numbers $\left\{\gamma_{k}\right\}_{k=1}^{\infty}$ as follows:

$$
\int_{\omega} \phi^{2}(x) d x \geq \gamma_{k}\|\phi\|_{L^{2}(\Omega)}^{2}, \quad \forall \phi \in L_{k}^{2}(\Omega) .
$$

Theorem 1.1: Approximate controllability in finite dimensions. Let

$$
\gamma_{k} \neq 0, k=1, \ldots
$$

(this condition is discussed in the next section). Given $T>0$, for cvery $K=1, \ldots, u_{0} \in L^{2}(\Omega), u_{1} \in L_{K}^{2}(\Omega), \varepsilon>0$ there are a control $v \in$ $L^{2}\left(Q_{T}\right)$ and $T^{*} \in[0, T]$ such that for all the corresponding to it (i.e., possibly multiple) solutions to $(1.1),(1.2)$

$$
\left\|\Pi_{K^{*}} u\left(\cdot, T^{*}\right)-u_{1}\right\|_{L^{2}(\Omega)} \leq \varepsilon
$$


where $\Pi_{K}$ stands for the operator of orthogonal projection in $L^{2}(\Omega)$ onto $L_{K}^{2}(\Omega)$.

Theorem 1.2: Approximate controllability property in $W$. Let (1.3) hold. Take any monotone decreasing sequence of positive numbers $\left\{c_{k}\right\}_{k=1}^{\infty}$ such that

$$
\lim _{k \rightarrow \infty} c_{k+1} / \gamma_{k}=0
$$

and define the space $W$ as in the above. Given $T>0$, for every $u_{0} \epsilon$ $L^{2}(\Omega), u_{1} \in W, \varepsilon>0$ there are a control $v \in L^{2}\left(Q_{T}\right)$ and $T^{*} \in[0, T]$ such that for all the corresponding to it (i.e., possibly multiple) solutions to $(1.1),(1.2)$

$$
\left\|u\left(\cdot, T^{*}\right)-u_{1}\right\|_{W} \leq \varepsilon
$$

In [15] E. Zuazua considered a semilinear heat equation with a superlinear locally Lipschitz term, also assuming that $f=f(u), f(0)=0$. By using the linearization of (1.1) around the zero-state and the implicit function theorem, he showed that the projections of its solutions on any finite dimensional space of $L^{2}(\Omega)$ can be steered to the zero-state (i.e., at an equilibrium) at any given in advance time, provided the initial datum is accordingly small enough. This property is regarded as the finite local exact null-controllability. (The local exact controllability of (1.1) with $u_{0} \in W_{\infty}^{1}(\Omega)$ and $f=f(x, t, u)$ was also studied in [5].) Our next result: Th. 1.3, can be viewed as an extension of this property to the global finite exact controllability, i.e., not necessarily at an equilibrium, see though Remark 1.1.4 below. (We, actually, do not assume that (1.1) has an equilibrium.) However, it should be noticed that Th. 1.3 deals specifically with the projections of solutions to (1.1), (1.2), (1.5) on the finite dimensional spaces spanned by the eigenfunctions $\omega_{k}$ 's only.

Theorem 1.3: Exact controllability in finite dimensions. Let all the conditions of Theorem 1.1 hold. In addition to that, assume that $f(x, t, u, p)=f(x, t, u)$ and for some positive constant $C_{*}$ :

$$
\begin{gathered}
\left|f\left(x, t, u_{1}\right)-f\left(x, t, u_{2}\right)\right| \leq C_{*}\left(\left|u_{1}\right|^{\alpha}+\left|u_{2}\right|^{\alpha}\right)\left|u_{1}-u_{2}\right| \\
\text { a.e. in } Q_{T}, \forall u_{1}, u_{2} \in R,
\end{gathered}
$$


where $\alpha=\left(2-q^{\prime}\right) /\left(2\left(q^{\prime}-1\right)\right)=2 / n$. Then, given $T>0$, for every $K=1, \ldots, u_{0} \in L^{2}(\Omega), u_{1} \in L_{K}^{2}(\Omega)$ there is a control $v \in L^{2}\left(Q_{T}\right)$ such that for the corresponding unique solution to (1.1), (1.2), (1.5)

$$
\Pi_{K} u(\cdot, T)=u_{1} .
$$

The result of Theorem 1.2 also holds at time $T$.

Remark 1:1: Discussion of main results.

- Due to the presence of multiple solutions, the classical definition of approximate controllability becomes ill-defined and, hence, does not apply to the boundary problem (1.1), (1.2). In that respect, we can view the fact that in Theorems 1.1 and 1.2 the instant $T^{*}$, when the desirable steering of (1.1) is achieved, is not necessarily equal to $T$ as a "payment" for the lack of uniqueness property. However, this circumstance does not seem essential to us, because, regardless of $u_{0}$ and $u_{1}, T^{*}$ is bounded by $T$.

- Furthermore, in the case when (1.1) possesses a unique solution, say, under the condition (1.5), the argument similar to that of Th. 1.3 yields that one can set $T^{*}=T$ in Th. 1.1 and 1.2. In this case the results of Th. 1.1 and $1.2 \mathrm{can}$, in fact, be viewed as the classical approximate controllability accordingly in every $\left(L_{K}^{2}(\Omega)\right)^{\prime}$ (if one regards $\left.L_{K}^{2}(\Omega) \subset L^{2}(\Omega)=\left(L^{2}(\Omega)\right)^{\prime} \subset\left(L_{K}^{2}(\Omega)\right)^{\prime}\right)$ and in $W$ but only from any $u_{0} \in L^{2}(\Omega)$. Accordingly, the result of Th. 1.3 can be interpreted as the classical exact controllability in every $H=\left(L_{K^{K}}^{2}(\Omega)\right)^{\prime}$ from an arbitrary $u_{0} \in L^{2}(\Omega)$.

- The strategy of the previous comment applies if one knows the exact position of the system "shortly" before the time $T$, see also the constructions of [6], p. 867. Unfortunately, if multiple solutions are an option, one cannot determine the actual state of the system merely from the knowledge of the initial state $u_{0}$ and the control applied. Hence, the application of the above strategy in the general case would require the introduction of so-called feedback control (i.e., when $v=v(x, t, u)$ ).

- It might seem worthy of noting that we do not require $\omega$ to be open, see also Ex. 1 in the next section. 
- It seems plausible that the assertions of Th. 1.1 and 1.3 on controllability in finite dimensions can be extended to a wider class of subspaces of $L^{2}(\Omega)$, not necessarily finite dimensional, namely those whose dual spaces in terms of the time-dependence satisfy an a priori estimate of type (3.7). In particular, the partial global approximate controllability to the subspace of $L^{2}(\Omega)$ generated by the functions vanishing on $\Omega \backslash \omega$ can be shown by using only the static (i.e., time-independent) controls.

\section{Discussion of condition (1.3)}

This assumption is very realistic. For example, it obviously holds for $\Omega_{*}=\Omega$ with $\gamma_{k}=1, k=1, \ldots$ Moreover, it was noticed in [11] that if $\partial \Omega$ is of class $C^{\infty}$ and $\Omega_{*}$ is open, then (1.3) holds with $\gamma_{k} \geq e^{-c \sqrt{\lambda_{k}}}, k=$ $1, \ldots$

We shall now consider two other examples for which (1.3) can be checked very easily.

Example 1. Let $n=1, \Omega=[0,1], \Omega_{*}$ be any subset of $[0,1]$ of positive measure, which has a limit point $x_{0}$ such that the corresponding sequences of points of $\Omega_{*}$ converging to $x_{0}$ form a set of positive measure in every neighborhood of $x_{0}$. Note that $\lambda_{k}=(\pi k)^{2}, \omega_{k}(x)=$ $\sqrt{2} \sin \pi k x, k=1 \ldots$

We argue by contradiction. Let us assume that (1.3) does not hold, i.e., some $\gamma_{K}=0$. By the finite dimension of $L_{K}^{2}(\Omega)$, one can distinguish a function $\phi_{*}(x)=\sum_{k=1}^{K} \beta_{k} \omega_{k}(x)$ such that:

$$
\left\|\phi_{*}\right\|_{L^{2}(\Omega)}=1, \quad \int_{\omega} \dot{\phi}_{*}^{2}(x) d x=0 .
$$

Let $z=x+i y$ be a complex variable. Since

$$
\omega_{k}(z)=\sqrt{2} \sin \pi k z=\sqrt{2} \frac{e^{i \pi k z}-e^{-i \pi k z}}{2 i}
$$

is analytic for every $k$, the function $\phi_{*}(z)$ will also be analytic in the domain $D=\{z=x+i y \mid-1 \leq y \leq 1, x \in[0,1]\}$. Our assumption on $\Omega_{*}$ guarantees that if $\int_{\omega} \phi_{*}^{2}(x) d x=0$, then $\phi_{*}(z)$ vanishes on some 
sequence of points having a limit point. The unique continuation property of analytic functions from its zero's, see, e.g., [14], p. 88, implies that $\phi_{*}(z)$ vanishes in $D$, which contradicts the first equality in (2.1). Hence, condition (1.3) holds.

Remark 2.1. Alternatively, we could take in the above any $\Omega_{*}$ whose limit points form a set of positive measure, e.g., the perfect set of positive measure of Cantor type, constructed on some subinterval of $[0,1]$.

This example can be extended to the case of rectangular-shaped $\Omega$ in $n$ dimensions.

Example 2: $\Omega$ strictly convex at the origin. Assume that $\lambda_{k}$ 's are simple and $w_{k}$ 's are of class $C^{1}$ and have piecewise continuously differentiable second derivatives. Let $\Omega_{*}$ be any open subset of $\Omega$. Assume that (1.3) does not hold, i.e., some $\gamma_{K}=0$. As in Ex. 1, due to the finite dimension of $L_{K}^{2}(\Omega)$, one can distinguish a function $\phi_{*}(x)=\sum_{k=1}^{K} \beta_{k} \omega_{k}(x)$ such that $(2.1)$ holds. Then,

$$
(\Delta)^{i} \phi_{*}=\sum_{k=1}^{K}\left(-\lambda_{k}\right)^{i}\left[\beta_{k} \omega_{k}(x)\right]=0 \quad \text { in } \Omega_{*}, \quad i=0,1, \ldots, K-1 .
$$

This system of linear algebraic equations in $\left[\beta_{k} \omega_{k}(x)\right]$ admits the zerosolution only. Hence,

$$
\beta_{k} \omega_{k}(x) \equiv 0 \quad \text { in } \omega, k=1, \ldots, K .
$$

If $\beta_{k} \neq 0$, then $\omega_{k}(x) \equiv 0$ in $\Omega_{*}$ and since,

$$
\left|\Delta \omega_{k}\right| \leq\left|\lambda_{k}\right|\left|\omega_{k}\right| \text { in } \Omega \text {, }
$$

Theorem 6 in [12], p. 99 implies that $\omega_{k}$ vanishes in $\Omega$. Hence, $\phi_{*} \equiv 0$ in $\Omega$, which contradicts to the first equality in (2.1).

\section{Observability and controllability of the trun- cated system}

Introduce the following truncated linear system:

$$
u_{L t}=\Delta u_{L}+v(x, t) \chi_{\Omega *}(x) \text { in } Q_{T},
$$




$$
\left.u_{L}\right|_{\Sigma_{T}}=0, \quad u_{L}(x, 0)=u_{0},
$$

where we used the subscript $L$ to indicate that (3.1) is, in fact, the linear trivial case of (1.1). Let

$$
\begin{gathered}
w_{t}=\Delta w \text { in } Q_{T} \\
\left.w\right|_{\Sigma_{T}}=0, \quad w(x, 0)=w_{0}(x) .
\end{gathered}
$$

It is well known that solutions to (3.1) and (3.2) admit the following representations:

$$
\begin{aligned}
u_{L}(x, t) & =\sum_{k=1}^{\infty} e^{-\lambda_{k} t} \int_{\Omega} u_{0}(r) \omega_{k}(r) d r \omega_{k}(x) \\
& +\sum_{k=1}^{\infty} \int_{0}^{t} e^{-\lambda_{k}(t-\tau)}\left(\int_{\Omega} v(r, \tau) \chi_{\Omega *}(r) \omega_{k}(r) d r\right) d \tau \omega_{k}(x) \\
& w(x, t)=\sum_{k=1}^{\infty} e^{-\lambda_{k} t} \int_{\Omega} w_{0}(r) \omega_{k}(r) d r \omega_{k}(x)
\end{aligned}
$$

where the series converge in the $L^{2}(\Omega)$-norm uniformly over $t \in[0, T]$.

For every $w_{0} \in L_{K^{\prime}}^{2}(\Omega)$ such that $\left\|w_{0}\right\|_{L^{2}(\Omega)}=1$ we have:

$$
\int_{0}^{T} \int_{\Omega_{*}} w^{2}(x, t) d x d t=\sum_{l, m=1}^{K} \frac{1-e^{-\left(\lambda_{l}+\lambda_{m}\right) T}}{\lambda_{l}+\lambda_{m}} \alpha_{l} \alpha_{m} \beta_{l m},
$$

where

$$
\beta_{l m}=\int_{\Omega} \omega_{l}(x) \omega_{m}(x) d x
$$

Since

$$
\lim _{T \rightarrow 0} \frac{1-e^{-\left(\lambda_{l}+\lambda_{m}\right) T}}{T\left(\lambda_{l}+\lambda_{m}\right)}=1, \quad \forall l, m
$$

we obtain

$$
\frac{1-e^{-\left(\lambda_{l}+\lambda_{m}\right) T}}{\lambda_{l}+\lambda_{m}}=T\left(1+\varepsilon_{l m}(T)\right), \quad \varepsilon_{l m}(T) \rightarrow 0 \text { as } T \rightarrow 0 .
$$


Let (1.3) hold. For every $K$ and $\left\|w_{0}\right\|_{L^{2}(\Omega)}=1$ we can write:

$$
\begin{gathered}
\int_{0}^{T} \int_{\Omega \cdot} w^{2}(x, t) d x d t=T \sum_{l, m=1}^{K} \alpha_{l} \alpha_{m} \beta_{l m}+T \varepsilon_{K}(T)= \\
T \int_{\Omega_{*}} w_{0}^{2}(x) d x+T \varepsilon_{K^{K}}(T) \geq T \gamma_{K}\left\|w_{0}\right\|_{L^{2}(\Omega)}^{2}+T \varepsilon_{K^{*}}(T)
\end{gathered}
$$

where $\varepsilon_{K^{*}}(T) \rightarrow 0$ as $T \rightarrow 0$. Select a $T(K)$ such that

$$
\left|\varepsilon_{K}(T)\right|<\gamma_{K} / 2, \quad \forall T \in(0, T(K)]
$$

Since we assumed in (3.5) and (3.6) that $\left\|w_{0}\right\|_{L^{2}(\Omega)}=1$, this gives

$$
\begin{aligned}
\int_{0}^{T} \int_{\Omega_{*}} w^{2}(x, t) d x d t & \geq \frac{\gamma_{K} T}{2}=\frac{\gamma_{K} T}{2}\left\|w_{0}\right\|_{L^{2}(\Omega)}^{2}, \forall w_{0} \in L_{K}^{2}(\Omega), \\
\left\|w_{0}\right\|_{L^{2}(\Omega)} & =1, \forall T \in(0, T(K)],
\end{aligned}
$$

which, by linearity, is tantamount to the following.

Lemma 3.1: Exact observability in finite dimensions. Let (1.3) hold. For every natural $K^{*}$ there is a $T(K)>0, T(K) \rightarrow 0$ as $K \rightarrow \infty$, such that

$$
\begin{aligned}
\int_{0}^{T} \int_{\Omega .} w^{2}(x, t) d x d t \geq & \frac{\gamma_{K} T}{2}\left\|w_{0}\right\|_{L^{2}(\Omega)}^{2}, \\
& \forall w_{0} \in L_{K}^{2}(\Omega), \quad \forall T \in(0, T(K)] .
\end{aligned}
$$

By the standard functional analysis duality argument (see, e.g., [1]), we can establish the following.

Lemma 3.2: Exact controllability in finite dimensions. Let (1.3) hold. Given natural $K$, let $T(K)$ be defined as in Lemma 3.1, see (3.6). For every $T \in(0, T(K)], u_{0} \in L^{2}(\Omega), u_{1} \in L_{K}^{2}(\Omega)$ there is a control 
$v \in L^{2}\left(Q_{T}\right)$ such that for the corresponding solution to (3.1) one has $\Pi_{K} u_{L}(\cdot, T)=u_{1}$ and

$$
\begin{gathered}
\|v\|_{L^{2}\left(Q_{T}\right)} \leq \sqrt{\frac{2}{\gamma_{K} T}}\left(\left\|u_{1}\right\|_{L^{2}(\Omega)}+\left\|\Pi_{K} u_{0}\right\|_{L^{2}(\Omega)}\right) \leq \\
\sqrt{\frac{2}{\gamma_{K} T}}\left(\left\|u_{1}\right\|_{L^{2}(\Omega)}+\left\|u_{0}\right\|_{L^{2}(\Omega)}\right) .
\end{gathered}
$$

Remark 3.1. The results on the exact controllability in finite dimensions are, in fact, obvious for the linear systems. What is of our concern here is the estimate (3.8), which is most crucial for our further study.

Proof. Fix $u_{0} \in L^{2}(\Omega), K$ and select any $u_{1} \in L_{K}^{2}(\Omega)$.

Recall now that the solutions of systems (3.1) with $u_{0}=0$ and (3.2) satisfy the well known duality relation:

$$
\int_{\Omega} w_{0}(x) u_{L}(x, T) d x=\int_{0}^{T} \int_{\Omega} w(x, T-t) v(x, t) d x d t, \forall w_{0} \in L_{K}^{2}(\Omega)
$$

which can be obtained by the multiplication of (3.1) with $u_{0}=0$ by $w(x, T-t)$ and further integration by parts over $Q_{T}$.

On the other hand, since

$$
\int_{0}^{T} \int_{\Omega .} w^{2}(x, T-t) d x d t=\int_{0}^{T} \int_{\Omega} w^{2}(x, t) d x d t
$$

(3.7) yields

$\left\|w_{0}\right\|_{L^{2}(\Omega)}^{2} \leq \frac{2}{\gamma_{K^{*} T}} \int_{0}^{T} \int_{\Omega} w^{2}(x, T-t) d z d t, \quad \forall w_{0} \in L_{K}^{2}(\Omega), \quad \forall T \in(0, T(K)]$.

This inequality defines a linear bounded operator, say,

$$
\begin{gathered}
\mathcal{R}_{K}(T): V_{K}(T) \ni \chi \Omega . w(\cdot, T-\cdot) \rightarrow w_{0} \in L_{K}^{2}(\Omega), \quad\left\|\mathcal{R}_{K}(T)\right\| \leq \sqrt{\frac{\overline{2}}{\gamma_{K} T}}, \\
V_{K}(T)=\left\{\chi \Omega . w(\cdot, T-t), t \in(0, T) \mid w_{0} \in L_{K}^{2}(\Omega)\right\},
\end{gathered}
$$


which maps the finite dimensional subspace $V_{K}(T)$ of $L^{2}\left(Q_{T}\right)$ onto $L_{K}^{2}(\Omega)$. Then, by the duality pairing between $V_{K}(T)$ and $L_{K}^{2}(\Omega)$,

$$
\begin{gathered}
\int_{\Omega} w_{0}(x) u_{1} d x=\left\langle w_{0}, u_{1}\right\rangle_{L_{K^{\prime}}^{2}(\Omega)}=\left\langle\mathcal{R}_{K}(T) \chi \Omega_{*} w(\cdot, T-\cdot), u_{1}\right\rangle_{L_{K^{2}}^{2}(\Omega)}= \\
<w(\cdot, T-\cdot), \mathcal{R}_{K^{*}}^{*}(T) u_{1}>_{L^{2}(\Omega \times \times(0, T))}= \\
\int_{0}^{T} \int_{\Omega_{*}} w(x, T-t) \mathcal{R}_{K^{*}}^{*}(T) u_{1} d x d t, \forall w_{0} \in L_{K^{\prime}}^{2}(\Omega)
\end{gathered}
$$

Hence, if we set, $v=\mathcal{R}_{K}^{*}(T) u_{1} \in V_{K}(T) \subset L^{2}\left(Q_{T}\right)$, where

$$
\mathcal{R}_{K^{*}}^{*}(T): L_{K^{\prime}}^{2}(\Omega) \rightarrow V_{K}(T), \quad\left\|\mathcal{R}_{K^{*}}^{*}(T)\right\| \leq \sqrt{\frac{2}{\gamma_{K} T}}, \quad \forall T \in(0, T(K)],
$$

then the comparison of the identities $(3,10)$ and $(3.9)$ on $L_{K}^{2}(\Omega)$ yields that this control generates $u_{L}(\cdot, T)$ such that $\Pi_{K} u_{L}(\cdot, T)=u_{1}$ and, in view of (3.11), the estimate (3.8) holds with $u_{0}=0$.

The general case follows from above from the presentation (3.3). Indeed, if $u_{0} \neq 0$, then to steer (3.1) at time $T$ to $u_{1}$ in the projection space $L_{K}^{2}(\Omega)$ one needs to apply the control which steers (3.1) from $u_{0}=0$ to $u_{1}-\sum_{k=1}^{K} e^{-\lambda_{k} T} \int_{\Omega} u_{0}(r) \omega_{k}(r) d r \omega_{k}(x)$ in this space.

\section{Proofs of Theorems 1.1 and 1.2}

Proof of Theorem 1.1. Fix $T>0, K, u_{0} \in L^{2}(\Omega), u_{1} \in L_{K^{-}}^{2}(\Omega)$.

Step 1. We will need the following estimates shown in [6], p. 865 that for solutions of (1.1), (1.2) and (3.1):

$$
\begin{aligned}
& \left\|u_{L}-u\right\|_{C\left(\left[0, T^{*}\right]_{i} L^{2}(\Omega)\right)} \leq c\left(T^{*}\right)\left\|\varphi^{*}\right\|_{L^{q^{*}}\left(Q_{\left.r^{*}\right)}\right.}+ \\
& c\left(T^{*}\right)\left[\text { meas }\{\Omega\} T^{*}\right]^{\frac{1}{q^{\prime}}\left(1-\frac{q^{*}}{q}\right)}\|u\|_{L^{q}\left(Q_{T^{*}}\right)}^{q^{*} / q^{\prime}}+
\end{aligned}
$$

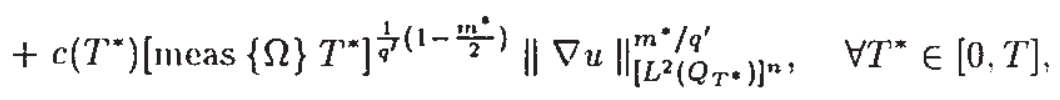


and

$$
\begin{gathered}
\|u\|_{\left.L^{q}\left(Q_{T^{*}}\right)\right)},\|u\|_{C\left(\left[0, T^{*}\right]_{i} L^{2}(\Omega)\right)}+\left(\int_{0} \int_{\Omega}\|\nabla u\|_{R^{n}}^{2} d x d t\right)^{1 / 2} \leq \\
c\left(T^{*}\right)\left(\left\|u_{0}\right\|_{L^{2}(\Omega)}+\sqrt{T^{*}}\|v\|_{L^{2}\left(Q_{T^{*}}\right)}+1\right), \quad \forall T^{*} \in[0, T],
\end{gathered}
$$

where $c(s), s>0$ is used to denote a generic positive non-decreasing function.

Combining these two inequalities yields (as in Th. 3.2 from [6], see also similar type of constructions in (5.13) below) that if for some $v_{\left(T^{*}\right)} \in L^{2}\left(Q_{T^{*}}\right)$

$$
\left(T^{*}\right)^{\frac{1}{2}+\min \left\{\frac{1}{q^{*}}\left(1-\frac{q^{*}}{q}\right), \frac{1}{m^{*}}\left(1-\frac{m^{*}}{2}\right)\right\}}\|v\|_{L^{2}\left(Q_{T^{*}}\right)} \rightarrow 0 \text { as } T^{*} \rightarrow 0,
$$

then for the corresponding solutions of (3.1) and (1.1), (1.2):

$$
\left\|u_{L}-u\right\|_{C\left(\left[0, T^{*}\right] ; L^{2}(\Omega)\right)} \rightarrow 0 \quad \text { as } T^{*} \rightarrow 0 .
$$

Step 2. Let the assumptions and notations of Lemma 3.1 hold. For every $T^{*} \in(0, T(K)] \cap[0, T]$, denote by $v_{\left(T^{*}\right)}$ the control in (3.8), which steers (3.1) at time $T^{*}$ from the given $u_{0}$ to the state $u_{L}\left(\cdot, T^{*}\right)$ whose projection on $L_{K}^{2}(\Omega)$ is $u_{1}$. By (3.8),

$$
\begin{gathered}
\left\|v_{\left(T^{*}\right)}\right\|_{L^{2}\left(Q_{\left.T^{*}\right)}\right.} \leq \sqrt{\frac{2}{\gamma_{K^{T}} T^{*}}}\left(\left\|u_{1}\right\|_{L^{2}(\Omega)}+\left\|u_{0}\right\|_{L^{2}(\Omega)}\right), \\
\forall T^{*} \in(0, T(K)] \bigcap[0, T] .
\end{gathered}
$$

Denote an arbitrary (if there are several of them) corresponding solution to $(1.1),(1.2)$ on $\left[0, T^{*}\right]$ by $u_{\left(T^{*}\right)}$. By $u_{L\left(T^{*}\right)}$ denote the corresponding solution of (3.1). Then, since (4.5) implies (4.3), the passage (4.4) holds for these solutions, that is,

$$
\left\|u_{L\left(T^{*}\right)}-u_{\left(T^{*}\right)}\right\|_{C\left(\left[0, T^{*}\right]_{i} L^{2}(\Omega)\right)} \rightarrow 0 \quad \text { as } T^{*} \rightarrow 0 .
$$

In particular, since $\Pi_{K^{*}} u_{L\left(T^{*}\right)}\left(\cdot, T^{*}\right)=u_{1}$ for all $T^{*} \in(0, T(K)] \cap[0, T]$ :

$$
\left\|\Pi_{K^{*}} u_{\left(T^{*}\right)}\left(\cdot, T^{*}\right)-u_{1}\right\|_{L^{2}(\Omega)} \rightarrow 0 \quad \text { as } T^{*} \rightarrow 0,
$$


which proves Th. 1.1.

\section{Proof of Theorem 1.2.}

Step 1. Fix $T>0, u_{0} \in L^{2}(\Omega)$ and consider an arbitrary $u_{1}=$ $\sum_{k=1}^{l} \alpha_{k} \omega_{k}$ lying in some $L_{K}^{2}(\Omega)$, i.e., $l \leq K$. Let the notations of the proof of Th. 1.1 hold.

Let now $K \rightarrow \infty$. Accordingly, $T(K) \rightarrow 0$, see Lemma 3.1 , so without loss of generality we can assume that $T(K) \leq T$. In its turn, from (4.6) it follows that for every $K>l$ there is a $t_{K} \in(0, T(K)]$ such that, say,

$$
\left\|\Pi_{K} u_{\left(t_{K}\right)}\left(\cdot, t_{K}\right)-u_{1}\right\|_{L^{2}(\Omega)} \leq \frac{1}{K}, K=l, l+1, \ldots .
$$

Hence, $u_{\left(t_{K}\right)}\left(\cdot, t_{K}\right)$ admits the following presentation:

$$
u_{\left(t_{K}\right)}\left(\cdot, t_{K}\right)=u_{K}^{(1)}+u_{K}^{(2)},
$$

where $u_{K}^{(1)}=\Pi_{K} u_{\left(t_{K}\right)}\left(\cdot, t_{K}\right)$ converge strongly in $L^{2}(\Omega)$ to $u_{1}$ as $K \rightarrow \infty$ and

$$
u_{K}^{(2)}=u_{\left(t_{K}\right)}\left(\cdot, t_{K}\right)-u_{K}^{(1)}, \quad u_{K}^{(1)} \perp u_{K}^{(2)} .
$$

Step 2. It follows from $(4.2)$ and $(3.8) /(4.5)$ that

$$
\begin{aligned}
& \left\|u_{\left(t_{K}\right)}\right\|_{C\left(\left[0, t_{K}\right] ; L^{2}(\Omega)\right)}+\left(\int_{0}^{t_{k}} \int_{\Omega}\left\|\nabla u_{\left(t_{K}\right)}\right\|_{R^{n}}^{2} d x d t\right)^{1 / 2} \leq \\
& c\left(t_{K}\right)\left(\left\|u_{0}\right\|_{L^{2}(\Omega)}+\sqrt{\frac{2}{\gamma_{K}}}\left(\left\|u_{0}\right\|_{L^{2}(\Omega)}+\left\|u_{1}\right\|_{L^{2}(\Omega)}\right)+1\right) \leq \\
& \frac{L\left(u_{0}, u_{1}\right)}{\sqrt{\gamma_{K}}}, K \geq l,
\end{aligned}
$$

where $L\left(u_{0}, u_{1}\right)$ is a positive number defined only by $u_{0}$ and $u_{5}$ (recall that $\gamma_{K}$ 's are non-increasing). Therefore,

$$
\left\|u_{K}^{(2)}\right\|_{L^{2}(\Omega)} \leq \frac{L\left(u_{0}, u_{1}\right)}{\sqrt{\gamma_{K}}}, \quad K \geq l
$$

Combining (1.4) with (4.7)-(4.9) yields:

$$
\left\|u_{\left(t_{K}\right)}\left(\cdot, t_{K}\right)-u_{1}\right\|_{W} \leq
$$




$$
\sqrt{c_{1}}\left\|u_{K}^{(1)}-u_{1}\right\|_{L^{2}(\Omega)}+\sqrt{c_{K+1}}\left\|u_{K}^{(2)}\right\|_{L^{2}(\Omega)} \rightarrow 0 \text { as } K \rightarrow \infty .
$$

Taking into account that the set of $u_{1}$ considered above is dense in $W$, this yields the assertion of Theorem 1.2 with $T^{*}$ to be one of the sufficiently small $t_{K}$ 's.

\section{Proof of Theorem 1.3}

First of all let us recall that the existence of a solution to (1.1), (1.2), (1.5) from the space $C\left([0, T] ; L^{2}(\Omega)\right) \cap H_{0}^{\mathrm{t}, 0}\left(Q_{T}\right) \cap L^{q}\left(Q_{T}\right)$ follows as in the above from [9], pp. 466-467. Its uniqueness is implied by the local Lipschitz property (1.5) (see, e.g., the estimate (5.9a) and Remark 5.2 below).

We will establish the following result first and then we will show that Lemma 5.1 implies Th. 1.3 .

Lemma 5.1. Let all the conditions of Theorem 1.3 hold. Then, given $T>0$, for every $K=1, \ldots, u_{0} \in L^{2}(\Omega), u_{1} \in L_{K}^{2}(\Omega)$ there are $a$ control $v \in L^{2}\left(Q_{T}\right)$ and $T^{*} \in[0, T]$ such that for the corresponding unique solution to (1.1), (1.2), (1.5)

$$
\Pi_{K} u\left(\cdot, T^{*}\right)=u_{1} .
$$

The result of Theorem 1.2 also holds.

\section{Proof of Lemma 5.1.}

\section{Remark 5.1.}

- To simplify the notations, in this section we (again) intensively use the generic symbols " $c$ " and " $c(s), s>0$ " to denote accordingly any (i.e., possibly different) positive constant and positive nondecreasing function. In each case, they can be evaluated precisely, but their values do not affect our main result.

- In the proof of Lemma 5.1 below we will use slightly different notations than in the above: we will assume that (1.1), (1.2) is considered on a fixed interval $[0, \hat{T}]$ (in place of $[0, T]$ ), while $T$ will range over $[0, \hat{T}]$. This change is to preserve the uniformity of constraints (1.2), regardless of the duration of the actua] control time involved. 
Fix a natural number $K$ and the target state $u_{1} \in L_{K}^{2}(\Omega)$, which, without loss of generality, we further assume to be a non-zero state. To simplify the calculations, first we will prove this lemma for $u_{0}=0$ and then in Step 7 we will demonstrate how it can be extended to the general case.

Step 1 . Denote by $S(t)$ the semigroup associated with (3.1). Then we can write

$$
u_{L}(\cdot, t)=\int_{0}^{t} S(t-\tau) v(\cdot, \tau) \chi \Omega_{\star} d \tau
$$

Given $K, T \in[0, \hat{T}] \cap(0, T(K)]$, recall from (3.11) that

$$
\begin{gathered}
\mathcal{R}_{K}^{*}(T): L_{K}^{2}(\Omega) \ni y \rightarrow v \in V_{K}(T) \subset L^{2}\left(Q_{T}\right), \\
\Pi_{k} \int_{0}^{T} S(T-\tau)\left(\mathcal{R}_{K}^{*}(T) y\right)(\cdot, \tau) \chi_{\Omega_{*}} d \tau=y \\
\left\|\mathcal{R}_{K}^{*}(T) y\right\|_{L^{2}\left(Q_{T}\right)} \leq \sqrt{\frac{2}{\gamma_{K} T}}\|y\|_{L^{2}(\Omega)} .
\end{gathered}
$$

Step 2. For every $T>0$, set from now on $v=v^{*}+v_{*}$, where $v^{*}=\mathcal{R}_{K}^{*}(T) u_{1} \in V_{K}(T)$, so that

$$
\Pi_{K} \int_{0}^{T} S(T-\tau) v^{*}(\cdot, \tau) \chi \Omega_{*} d \tau=u_{1}
$$

while $v_{*}$ ranges over $V_{K}(T)$. (This presentation will be used below to introduce the equation (5.10) to implement the fixed-point argument.) Then we can write for $t \in[0, T]$ :

$$
\begin{aligned}
u_{L}=u_{L *}+u_{*}, \quad u_{L *}(\cdot, t) & =\int_{0}^{t} S(t-\tau) v^{*}(\cdot, \tau) \chi \Omega_{*} d \tau, \quad \Pi_{K^{*}} u_{L^{*}}(\cdot, T)=u_{1}, \\
u_{*}(\cdot, t) & =\int_{0}^{t} S(t-\tau) v_{*}(\cdot, \tau) \chi \Omega_{*} d \tau .
\end{aligned}
$$


Denote $z=u-u_{L}$. Then

$$
u=u_{L *}+u_{*}+z=u_{L *}+u_{*}+\mathcal{Q}(y),
$$

where, given $v^{*}$ and $K$, we introduced the nonlinear operator $\mathcal{Q}: y \rightarrow z$ as follows:

$$
\begin{aligned}
\mathcal{Q}: & L_{K}^{2}(\Omega) \ni y \rightarrow z=\mathcal{Q}(y)= \\
& \quad-\int_{0}^{t} S(t-\tau)(f(\cdot, \cdot u))(\cdot, \tau) d \tau \in C\left([0, T] ; L^{2}(\Omega)\right),
\end{aligned}
$$

where $u$ is the solution to (1.1), (1.2), (1.5), generated by $v^{*}+v_{*}, v_{*}=$ $\mathcal{R}_{K}^{*}(T) y$. We also set

$$
\begin{aligned}
\mathcal{Q}_{T} & :\left.L_{K}^{2}(\Omega) \ni y \rightarrow z\right|_{t=T}=\left.\mathcal{Q}(y)\right|_{t=T}=\mathcal{Q}_{T}(y)= \\
& -\int_{0}^{T} S(T-\tau)(f(\cdot, \cdot, u))(\cdot, \tau) d \tau \in L^{2}(\Omega) .
\end{aligned}
$$

Step 3. Given $v^{*}$, let us show that these operators are continuous on the following bounded subset of $L_{K^{-}}^{2}(\Omega)$ :

$U_{d}=\left\{w \mid w \in L_{K^{\prime}}^{2}(\Omega),\|w\|_{L^{2}(\Omega)} \leq d\right\}, \quad d=\left\|\Pi_{K^{u} u_{L *}}(\cdot, T)\right\|_{L^{2}(\Omega)}=\left\|u_{1}\right\|_{L^{2}(\Omega)}$, provided $T$ is small enough $\left(T=T\left(U_{d}\right)\right)$. It is sufficient to do this for the operator $\mathcal{Q}$ only.

Indeed, take any $y_{* *}, y_{*} \in U_{d}$. Then denote by $u_{* *}$ and $u_{*}$ the solutions to (3.1) (remind, with $u_{0}=0$ ), generated accordingly by

$$
v=v_{* *}=\mathcal{R}_{K}^{*}(T) y_{* *}, \quad v=v_{*}=\mathcal{R}_{K}^{*}(T) y_{*},
$$

in which case, in view of $(5.2), \Pi_{K} u_{* *}(\cdot, T)=y_{* *}, \Pi_{K^{*}} u_{*}(\cdot, T)=y_{*}$. By employing the Lipschitz property (1.5), from the classical energy estimate [9], p. 139, we obtain:

$$
\begin{gathered}
\left\|\mathcal{Q}\left(y_{* *}\right)-\mathcal{Q}\left(y_{*}\right)\right\|_{C\left([0, T] ; L^{2}(\Omega)\right)} \\
\leq c(T)\left(\int _ { 0 } ^ { T } \int _ { \Omega } \left(\left(f\left(x, t, u\left(u_{* *}\right)\right)-f\left(x, t, u\left(u_{*}\right)\right)^{q^{\prime}} d x d \tau\right)^{1 / q^{\prime}} \leq\right.\right.
\end{gathered}
$$




$$
c(T) C_{*}\left(\int_{0}^{T} \int_{\Omega}\left|u\left(u_{* *}\right)-u\left(u_{*}\right)\right|^{q^{\prime}}\left(\left|u\left(u_{* *}\right)\right|^{\alpha}+\left|u\left(u_{*}\right)\right|^{\alpha}\right)^{q^{\prime}} d x d \tau\right)^{1 / q^{\prime}}
$$

where $u\left(u_{* *}\right)$ and $u\left(u_{*}\right)$ are the solutions to (1.1), (1.2), (1.5) corresponding to $u_{* *}$ and $u_{*}$. Applying (twice) Hölder's inequality then yields:

$$
\begin{aligned}
& \left\|\mathcal{Q}\left(y_{* *}\right)-\mathcal{Q}\left(y_{*}\right)\right\|_{C\left([0, T] ; L^{2}(\Omega)\right)} \leq \\
& c(T)\left\|u\left(u_{* *}\right)-u\left(u_{*}\right)\right\|_{C\left([0, T] ; L^{2}(\Omega)\right)}\left(\int _ { 0 } ^ { T } \left(\int _ { \Omega } \left(\|\left. u\left(u_{* *}\right)\right|^{\alpha}+\right.\right.\right. \\
& \left.\left.\left|u\left(u_{*}\right)\right|^{\alpha}\right)\left.^{\frac{2 q^{\prime}}{2-q^{\prime}}} d x\right|^{\frac{2-q^{\prime}}{2}} d \tau\right)^{1 / q^{\prime}} \leq \\
& c(T) T^{1 / 2}\left\|u\left(u_{* *}\right)-u\left(u_{*}\right)\right\|_{C\left([0, T]_{i} L^{2}(\Omega)\right)}\left(\int _ { 0 } ^ { T } \int _ { \Omega } \left(\left|u\left(u_{* *}\right)\right|^{\alpha}+\right.\right. \\
& \left.\left.\left|u\left(u_{*}\right)\right|^{\alpha}\right)^{\frac{2 q^{\prime}}{2-q^{\prime}}} d x d \tau\right)^{\frac{2-q^{\prime}}{2 q^{\prime}}}
\end{aligned}
$$

(see Remark 5.1.1). From here, by using (twice) the inequality $(a+b)^{\gamma} \leq$ $C\left(a^{\gamma}+b^{\gamma}\right), \forall a, b, \gamma \geq 0$ for some $C=C(\gamma)>0$, we derive:

$$
\begin{gathered}
\left\|\mathcal{Q}\left(y_{* *}\right)-\mathcal{Q}\left(y_{*}\right)\right\|_{C\left([0, T] ; L^{2}(\Omega)\right)} \leq \\
c(T) T^{1 / 2}\left\|u\left(u_{* *}\right)-u\left(u_{*}\right)\right\|_{C\left([0, T]_{i} L^{2}(\Omega)\right)}\left(\left\|u\left(u_{* *}\right)\right\|_{L^{\frac{2 q q^{\prime}}{2-q^{\prime}}\left(Q_{T}\right)}}^{\alpha}+\right. \\
\left.\left\|u\left(u_{*}\right)\right\|_{L^{\frac{2 \times q^{\prime}}{2-q^{\prime}}\left(Q_{T}\right)}}^{\alpha}\right)
\end{gathered}
$$

Since $\alpha=\left(2-q^{\prime}\right) / 2\left(q^{\prime}-1\right)$,

$$
\frac{2 \alpha q^{\prime}}{2-q^{\prime}}=q
$$

and (5.4) becomes

$$
\begin{gathered}
\left\|\mathcal{Q}\left(y_{* *}\right)-\mathcal{Q}\left(y_{*}\right)\right\|_{C\left([0, T] ; L^{2}(\Omega)\right)} \leq \\
c(T) T^{1 / 2}\left\|u\left(u_{* *}\right)-u\left(u_{*}\right)\right\|_{\left.C\left([0, T] ; L^{2}(\Omega)\right)\right\}}\left(\left\|u\left(u_{* *}\right)\right\|_{L^{q}\left(Q_{T}\right)}^{\alpha}\right. \\
\left.+\left\|u\left(u_{*}\right)\right\|_{L^{q}\left(Q_{T}\right)}^{\alpha}\right) .
\end{gathered}
$$


On the other hand, it follows from (4.2) that

$$
\begin{gathered}
\left\|u\left(u_{* *}\right)\right\|_{L^{q}\left(Q_{T}\right)} \leq c(T)\left(1+T^{1 / 2}\left\|v^{*}\right\|_{L^{2}\left(Q_{T}\right)}+T^{1 / 2}\left\|v_{* *}\right\|_{L^{2}\left(Q_{T}\right)}\right), \\
\left\|u\left(u_{*}\right)\right\|_{L^{q}\left(Q_{T}\right)} \leq c(T)\left(1+T^{1 / 2}\left\|v^{*}\right\|_{L^{2}\left(Q_{T}\right)}+T^{1 / 2}\left\|v_{*}\right\|_{L^{2}\left(Q_{T}\right)}\right) .
\end{gathered}
$$

By $(5.2) /(3.8)$, this results in

$$
\begin{aligned}
& \left\|u\left(u_{* *}\right)\right\|_{L^{q}\left(Q_{T}\right)}^{\alpha}+\left\|u\left(u_{*}\right)\right\|_{L^{q}\left(Q_{T}\right)}^{\alpha} \leq
\end{aligned}
$$

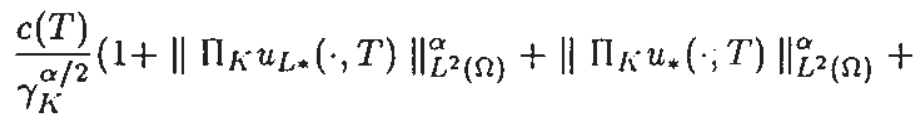

$$
\begin{aligned}
& \left.\left\|I l_{K} u_{* *}(\cdot, T)\right\|_{L^{2}(\Omega)}^{\alpha}\right)
\end{aligned}
$$

(see Remark 5.1.1). Combining (5.6) and (5.5) gives

$$
\begin{gathered}
\left\|\mathcal{Q}\left(y_{* *}\right)-\mathcal{Q}\left(y_{*}\right)\right\|_{C\left([0, T]_{;} L^{2}(\Omega)\right)} \leq \\
\frac{c(T) T^{1 / 2}}{\gamma_{K}^{\alpha / 2}}\left\|u\left(u_{* *}\right)-u\left(u_{*}\right)\right\|_{C\left([0, T] ; L^{2}(\Omega)\right)}\left(1+\left\|\Pi_{K} u_{L *}(\cdot, T)\right\|_{L^{2}(\Omega)}^{\alpha}+\right. \\
\left.\left\|\Pi_{K} u_{*}(\cdot, T)\right\|_{L^{2}(\Omega)}^{\alpha}+\left\|\Pi_{K} u_{* *}(\cdot, T)\right\|_{L^{2}(\Omega)}^{\alpha}\right)
\end{gathered}
$$

Assume now that, given $U_{d}, T$ is such a small number that

$$
\begin{gathered}
\frac{c(T) T^{1 / 2}}{\gamma_{K}^{\alpha / 2}}\left(1+\left\|\Pi_{K^{\prime} u_{L *}}(\cdot, T)\right\|_{L^{2}(\Omega)}^{\alpha}+\left\|\Pi_{K} u_{*}(\cdot, T)\right\|_{L^{2}(\Omega)}^{\alpha}+\right. \\
\left.\left\|\Pi_{K^{\prime} u_{* *}}(\cdot, T)\right\|_{L^{2}(\Omega)}^{\alpha}\right) \leq \\
\frac{c(T) T^{1 / 2}}{\gamma_{K^{\alpha}}^{\alpha / 2}}\left(1+3 d^{\alpha}\right) \leq \frac{1}{2}
\end{gathered}
$$

(Recall that $\Pi_{K^{*} u_{* *}}(\cdot, T)=y_{* *}, \Pi_{K^{*} u_{*}}(\cdot, T)=y_{*}$ ) Then, since by $(5.3 \mathrm{~b})$, applied for $y_{*}$ and $y_{* * *}$,

$$
\begin{gathered}
\left\|u\left(u_{* *}\right)-u\left(u_{*}\right)\right\|_{C\left([0, T] ; L^{2}(\Omega)\right)} \leq\left\|u_{* * *}-u_{*}\right\|_{C\left(\left[0, T^{7}\right] ; L^{2}(\Omega)\right\}}+ \\
\left\|\mathcal{Q}\left(y_{* *}\right)-\mathcal{Q}\left(y_{*}\right)\right\|_{C\left(\{0, T] ; L^{2}(\Omega)\right)}
\end{gathered}
$$

combining the latter and (5.7) yields

$$
\left\|\mathcal{Q}\left(y_{* *}\right)-\mathcal{Q}\left(y_{*}\right)\right\|_{C\left([0, T] ; L^{2}(\Omega)\right)} \leq \frac{1}{2}\left\|u\left(u_{* *}\right)-u\left(u_{*}\right)\right\|_{C\left([0, T] ; L^{2}(\Omega)\right)} \leq
$$


$\frac{1}{2}\left\|u_{* *}-u_{*}\right\|_{C\left([0, T] ; L^{2}(\Omega)\right)}+\frac{1}{2}\left\|\mathcal{Q}\left(y_{* *}\right)-\mathcal{Q}\left(y_{*}\right)\right\|_{C\left([0, T]_{i} L^{2}(\Omega)\right)}$

or

$$
\left\|\mathcal{Q}\left(y_{* *}\right)-\mathcal{Q}\left(y_{*}\right)\right\|_{C\left([0, T] ; L^{2}(\Omega)\right)} \leq\left\|u_{* *}-u_{*}\right\|_{C\left([0, T] ; L^{2}(\Omega)\right)},
$$

and further, along with the standard energy estimate for the linear heat equation,

$$
\begin{aligned}
\left\|\mathcal{Q}\left(y_{* *}\right)-\mathcal{Q}\left(y_{*}\right)\right\|_{C\left([0, T]_{;} L^{2}(\Omega)\right)} & \leq\left\|u_{* *}-u_{*}\right\|_{C\left([0, T] ; L^{2}(\Omega)\right)} \leq \\
2 T^{1 / 2} \| v_{* *}-v_{*} & \|_{L^{2}\left(Q_{T}\right)}= \\
2 T^{1 / 2}\left\|\mathcal{R}_{K}^{*}(T)\left(y_{* *}-y_{*}\right)\right\|_{L^{2}\left(Q_{T}\right)} & \leq 2 \sqrt{\frac{2}{\gamma_{K}}}\left\|y_{* *}-y_{*},\right\|_{L^{2}(\Omega),}
\end{aligned}
$$

implying the desirable continuity of $\mathcal{Q}$ and $\mathcal{Q}_{T}$ on $U_{d}$. Denote an arbitrary continuous extension of the operator $\mathcal{Q}_{T}$ from $U_{d}$ to $L_{K}^{2}(\Omega)$ by $\bar{Q}_{T d}$.

Remark 5.2. If one considers any two solutions $u_{(1)}$ and $u_{(2)}$ to (1.1), (1.2), (1.5) (i.e., not necessarily as those in Step 3), then the estimate (5.5) holds for them as well. It can be used to prove the uniqueness of solutions to this system. Indeed, let $u_{(1)}$ and $u_{(2)}$ be generated by the same initial condition and control. Select $T_{1}$ such that

$$
c(\bar{T}) T_{1}^{1 / 2}\left(\left\|u_{(1)}\right\|_{L^{q}\left(Q_{T}\right)}^{\alpha}+\left\|u_{(2)}\right\|_{L^{q}\left(Q_{T}\right)}^{\alpha}\right) \leq \frac{1}{2} .
$$

Then (5.5), applied for $T=T_{1}$, yields

$$
\left\|u_{(1)}-u_{(2)}\right\|_{C\left(\left[0, T_{1}\right] ; L^{2}(\Omega)\right)} \leq \frac{1}{2}\left\|u_{(1)}-u_{(2)}\right\|_{C\left(\left[0, T_{1}\right] ; L^{2}(\Omega)\right)},
$$

which implies that $u_{(1)}=u_{(2)}$ on $\left[0, T_{1}\right]$. Since the norms on the right in the above inequality can be evaluated solely in terms of the initial data and control (sce (4.2)), tepeating this argument finitely many times gives us uniqueness on $[0, \tilde{T}]$.

Step 4. Given $v^{*}$, consider the following equation in $L_{K}^{2}(\Omega)$ :

$$
y+\Pi_{K} \overline{\mathcal{Q}}_{\tau d}(y)=0 .
$$


Since $L_{K}^{2}(\Omega)$ is finite dimensional, the continuity of the operator $\Pi_{K} \mathcal{Q}_{T d}$ : $U_{d} \rightarrow L_{K^{\prime}}^{2}(\Omega)$, established in Step 3 under condition (5.8), implies its compactness as well. Assuming (5.8), i.e., that $T$ is sufficiently small, by Altman's fixed point theorem, see [13], p. 97 this equation has a solution if for some constant $L(K, T)$ the following estimate holds:

$$
\begin{gathered}
\left\|\Pi_{K} \overline{\mathcal{Q}}_{T d}(y)+y\right\|_{L^{2}(\Omega)}^{2} \geq\left\|\Pi_{K} \overline{\mathcal{Q}}_{T^{d}}(y)\right\|_{L^{2}(\Omega)}^{2}-\|y\|_{L^{2}(\Omega)}^{2} \\
\forall y \in L_{K}^{2}(\Omega):\|y\|_{L^{2}(\Omega)}=L(K, T) .
\end{gathered}
$$

The $L^{2}(\Omega)$-norm of this solution does not exceed this $L(K, T)$. It suffices then to establish the existence of such an $L(K, T)$, for which the expression on the right in (5.11) is non-positive. To this end, set

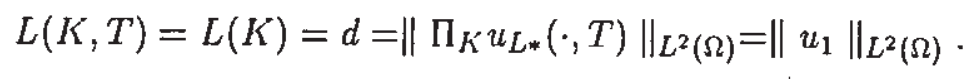

Then (4.1) and (4.2) with $m^{*}=0$, combined with (5.2), give for $\|y\|_{L^{2}(\Omega)}=d=L(K)$ :

$$
\begin{aligned}
& \left\|\left.\left(u-u_{L}\right)\right|_{t=T}\right\|_{L^{2}(\Omega)}=\left\|\overline{\mathcal{Q}}_{T d}(y)\right\|_{L^{2}(\Omega)}=\left\|\mathcal{Q}_{T}(y)\right\|_{L^{2}(\Omega)} \leq \\
& c(T)\left(\left\|\varphi^{*}\right\|_{L^{q^{\prime}}\left(Q_{T}\right)}+\right. \\
& T^{\frac{1}{q^{(1}}\left(1-\frac{q^{*}}{q}\right)}\left(1+\left(\frac{\left\|\Pi_{K} u_{L *}(\cdot, T)\right\|_{L^{2}(\Omega)}+\|y\|_{L^{2}(\Omega)}}{\gamma_{K}^{1)^{2}}}\right)^{q^{*} / q^{\prime}}\right) \leq
\end{aligned}
$$

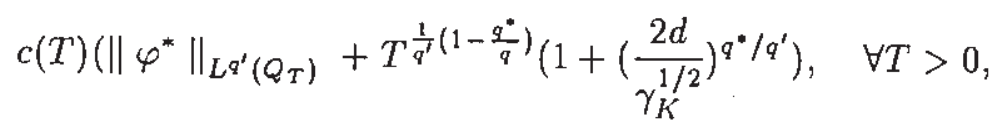

see Remark 5.1.1. Then (5.13) implies the existence of $T$, which satisfies (5.8) with this $d$ and, simultaneously,

$$
\begin{gathered}
\left\|\Pi_{K} \overline{\mathcal{Q}}_{T d}(y)\right\|_{L^{2}(\Omega)} \leq\left\|\overline{\mathcal{Q}}_{T d}(y)\right\|_{L^{2}(\Omega)}=\left\|\mathcal{Q}_{T}(y)\right\|_{L^{2}(\Omega)} \leq d=L(K), \\
\forall y \in L_{K}^{2}(\Omega):\|y\|_{L^{2}(\Omega)}=d=L(K) .
\end{gathered}
$$

Therefore, $(5.10)$ has a solution $\hat{y} \in L_{K}^{2}(\Omega)$, whose norm does not exceed $d$, which means that

$$
\hat{y}=-\Pi_{K} \overline{\mathcal{Q}}_{T d}(\hat{y})=-\Pi_{K} \mathcal{Q}_{T}(\hat{y}) .
$$


Step 5. (5.15) means that there is a $v_{*}=\mathcal{R}_{K}^{*}(T) \hat{y}$ such that for the corresponding solution $u_{*}$ to $(3.1)$ one has $\hat{y}=\Pi_{K} u_{*}(\cdot, T)=-\Pi_{K} \mathcal{Q}_{T}(\hat{y})$. Hence, by (5.3),

$$
u(\cdot, T)=u_{L *}(\cdot, T)+\hat{y}+\mathcal{Q}_{T}(\hat{y}),
$$

which implies $(1.6)^{\prime}$ in Lemma 5.1 with $T^{*}=T$, provided $T \in$ $[0, \hat{T}] \cap(0, T(K)]$ is small enough to satisfy $(5.8)$ and $(5.13),(5.14)$. Since $u_{1}$ was selected arbitrarily in $L_{K}^{2}(\Omega)$, this yields the exact controllability of (1.1), (1.2), (1.5) in $L_{K^{\prime}}^{2}(\Omega)$ from $u_{0}=0$ as described in Lemma 5.1 .

Step 6. Based on estimates (5.12) and (5.2), the argument of Th. 1.2 applies to the system $(1.1),(1.2),(1.5)$, which yields the second part of Lemma 5.1.

This completes the proof of Lemma 5.1 in the case when $u_{0}=0$.

Step 7. All the above results hold true if in (1.1), (1.2), (1.5) and (3.1) we set $\left.u\right|_{t=0}=\left.u_{L}\right|_{t=0}=u_{0}, \forall u_{0} \in L^{2}(0,1)$. Indeed, the most essential correction to be made in this case in the above argument is the replacement of $u_{L *}$ in (5.3) by

$$
u_{L *}(\cdot, t)=S(t) u_{0}+\int_{0}^{t} S(t-\tau) v^{*}(\cdot, \tau) \chi \Omega_{*} d \tau
$$

This completes the proof of Lemma 5.1.

Proof of Theorem 1.3. The idea of the proof of Lemma 5.1 is to apply the actual control only on the time-interval $\left[0, T^{*}\right]$, when $T^{*}$ is sufficiently small. On the other hand, due to the time-invariance of the systems (3.1)/(3.2) providing the key estimates $(3.7) /(3.8)$, only the length of the time-interval really matters here. Hence, the same technique can be applied for the intervals $\left[T_{*}, T\right]$ with $T_{*} \rightarrow T$, where $T$ is given, while treating $u\left(\cdot, T_{*}\right)$ as a new initial datum. Accordingly, on $\left[0, T_{*}\right]$ one can use, say, the zero-control, which insures by (4.2) the uniform boundedness of these $u\left(\cdot, T_{*}\right)$. This completes the proof of Theorem 1.3. 
Remark 5.3. Let us note that the major technical reason for the introduction of the assumption (1.5) in Lemma 5.1/Theorem 1.3 is the necessity to prove the continuity of the operator $\mathcal{Q}_{T}$ in Step 3. (The chain of inequalities (5.13) remain true under the conditions (1.2) even if one uses the full versions of the a priori estimates (4.1) and (4.2), i.e., with convection). Therefore, it seems possible to extend Th. 1.3 to some cases of semilinear reaction-convection terms, for which: a) (1.1) admits a unique solutions, and $b$ ) the just-mentioned continuity takes place.

\section{References}

[1] Sz. Dolecki and D.L. Russell (1977), A general theory of observation and control, SIAM J. Control and Opt, 15, pp. 185-219.

[2] C. Fabre, J.-P. Puel and E. Zuazua, Approximate controllability for the semilinear heat equations, Proc. Royal Soc, Edinburg, 125A (1995), pp. 31-61.

[3] E. Fernandez-Cara, Null controllability for semilinear heat equation, ESAIM: Control, Optimization and Calculus of Variations, (1997), pp. 87-103.

[4] L. A. Fernández and E. Zuazua, Approximate controllability of the semilinear heat equation via optimal control, Preprint Universidad de Cantabria, 1997.

[5] A. Fursikov and O. Imanuvilov, Controllability of evolution equations, Lect. Note Series 34, Res. Inst. Math., GARC, Seoul National University, 1996.

[6] A.Y. Khapalov, Some aspects of the asymptotic behavior of the solutions of the semilinear heat equation and approximate controllability, J. Math. Anal. Appl., 194 (1995), pp. 858-882.

[7] A.Y. Khapalov, Approximate controllability and its well-posedness for the semilinear reaction-diffusion equation with internal lumped controls, ESAIM: $C O C V$, v. 3 (1999), p. 82. 
[8] A.Y. Khapalov, Exact null-controllability for the semilinear heat equation with superlinear nonlinear term and mobile internal controls, to appear in Nonlinear Analysis: TMA.

[9] O. H. Ladyzhenskaja, V.A. Solonnikov and N.N. Ural'ceva, "Linear and Quasi-linear Equations of Parabolic Type," AMS, Providence, Rhode Island, 1968.

[10] J.-L. Lions, Remarques sur la contrôlabilité approcheé. Proceedings of "Jornadas Hispano-Francesas sobre Control de Sistemas Distribuidos", University of Málaga, Spain, October 1990.

[11] J.-L. Lions and E. Zuazua, The cost of controlling unstable systems: time irreversible systems, Rev. Mat. Complutense, 10 1997, pp. 481523.

[12] L. Nirenberg, Uniqueness in Cauchy problems for differential equations with constant leading coefficients, Comm. Pure Appl. Math. 10 (1957), pp. 89-105.

[13] J.T. Schwartz, Nonlinear Functional Analysis, Notes on Mathematics and its applications, Gordon and Breach, New-York, 1969.

[14] E.C. Titchmarsh, The Theory of Functioms, Oxford University Press, Second Edition.

[15] E. Zuazua, Finite dimensional null controllability for the semilinear heat equation, J. Math. Pures et Appl., 76 (1997), pp. 237-264.

Department of Pure and Applied Mathematics

Washington State University, Pullman, WA 99164-3113;

fax: (509) 3351188 ;

tel. (509) 3353172 ;

$e$-mail: khapalaodelta.math.wsu.edu

Recibido: 15 de Octubre de 1997

Revisado: 22 de Marzo de 1999 